\title{
Article
}

\section{Book graphs are cycle antimagic}

\author{
Muhammad Awais Umar, ${ }^{1}$, Noshad $\mathrm{Ali}^{2}$, Afshan Tabassum ${ }^{2}$ and Basharat Rehman Ali ${ }^{3}$ \\ 1 Government Degree College (B), Sharaqpur Shareef, Pakistan. \\ 2 Department of Mathematics, NCBA\&E, DHA Campus, Lahore, Pakistan.; noshadaliue@gmail.com(N.A); \\ afshintabassum@gmail.com(A.T) \\ 3 Abdus Salam School of Mathematical Sciences, GC University, Lahore.; basharatrwp@gmail.com \\ * Correspondence: owais054@gmail.com
}

Received: 12 March 2019; Accepted: 1 Jun 2019; Published: 30 Jun 2019.

\begin{abstract}
Let $G=(V, E)$ be a finite simple graph with $v=|V(G)|$ vertices and $e=|E(G)|$ edges. Further suppose that $\mathbb{H}:=\left\{H_{1}, H_{2}, \ldots, H_{t}\right\}$ is a family of subgraphs of $G$. In case, each edge of $E(G)$ belongs to at least one of the subgraphs $H_{i}$ from the family $\mathbb{H}$, we say $G$ admits an edge-covering. When every subgraph $H_{i}$ in $\mathbb{H}$ is isomorphic to a given graph $H$, then the graph $G$ admits an $H$-covering. A graph $G$ admitting $H$ covering is called an $(a, d)$-H-antimagic if there is a bijection $\eta: V \cup E \rightarrow\{1,2, \ldots, v+e\}$ such that for each subgraph $H^{\prime}$ of $G$ isomorphic to $H$, the sum of labels of all the edges and vertices belongs to $H^{\prime}$ constitutes an arithmetic progression with the initial term $a$ and the common difference $d$. For $\eta(V)=\{1,2,3, \ldots, v\}$, the graph $G$ is said to be super $(a, d)$-H-antimagic and for $d=0$ it is called $H$-supermagic. When the given graph $H$ is a cycle $C_{m}$ then $H$-covering is called $C_{m}$-covering and super $(a, d)$-H-antimagic labeling becomes super $(a, d)-C_{m}$-antimagic labeling. In this paper, we investigate the existence of super $(a, d)-C_{m}$-antimagic labeling of book graphs $B_{n}$, for $m=4, n \geq 2$ and for differences $d=1,2,3, \ldots, 13$.
\end{abstract}

Keywords: Book graph $B_{n}$, super $(a, d)-C_{4}$-antimagic.

MSC: 05C78, 05C70.

\section{Introduction}

$\mathbf{L}$ et $G$ be a finite and simple graph. A family of subgraphs $H_{1}, H_{2}, \ldots, H_{t}$ is defined as an edge-covering of $G$ such that each edge of $E(G)$ belongs to at least one of the subgraphs $H_{i}, i=1,2, \ldots, t$. Then $G$ admits an $\left(H_{1}, H_{2}, \ldots, H_{t}\right)$-(edge) covering. If every subgraph $H_{i}$ is isomorphic to a given graph $H$, then the graph $G$ admits an $H$-covering. A graph $G$ admitting an $H$-covering is called $(a, d)$ - $H$-antimagic if there exists a total labeling $\eta: V(G) \cup E(G) \rightarrow\{1,2, \ldots, v+e\}$ such that for each subgraph $H^{\prime}$ of $G$ isomorphic to $H$, the $H^{\prime}$-weights,

$$
w t_{\eta}\left(H^{\prime}\right)=\sum_{v \in V\left(H^{\prime}\right)} \eta(v)+\sum_{e \in E\left(H^{\prime}\right)} \eta(e),
$$

constitute an arithmetic progression $a, a+d, a+2 d, \ldots, a+(t-1) d$, where $a>0$ and $d \geq 0$ are two integers and $t$ is the number of all subgraphs of $G$ isomorphic to $H$. Moreover, $G$ is said to be super $(a, d)$-H-antimagic, if the smallest possible labels appear on the vertices. If $G$ is a super $(a, d)$-H-antimagic graph then the corresponding total labeling $\eta$ is called the super $(a, d)$-H-antimagic labeling. For $d=0$, the super $(a, d)$ - $H$-antimagic graph is called $H$-supermagic.

The $H$-supermagic graph was first introduced by Gutiérrez et al. in [1]. They proved that the star $K_{1, n}$ and the complete bipartite graphs $K_{n, m}$ are $K_{1, h}$-supermagic for some $h$. They also proved that the path $P_{n}$ and the cycle $C_{n}$ are $P_{h}$-supermagic for some $h$. Lladó et al. [2] investigated $C_{n}$-supermagic graphs and proved that wheels, windmills, books and prisms are $C_{h}$-magic for some $h$. Some results on $C_{n}$-supermagic labelings of several classes of graphs can be found in [3]. Maryati et al. [4] gave $P_{h}$-supermagic labelings of shrubs, subdivision of shrubs and banana tree graphs. Other examples of $H$-supermagic graphs with different choices of $H$ have been given by Jeyanthi et al. in [5]. Maryati et al. [6] investigated the $G$-supermagicness of a disjoint union of $c$ copies of a graph $G$ and showed that disjoint union of any paths is $c P_{h}$-supermagic for some $c$ and $h$.

The $(a, d)$ - $H$-antimagic labeling was introduced by Inayah et al. [7]. In [8] Inayah et al. investigated the super $(a, d)$-H-antimagic labelings for some shackles of a connected graph $H$. 
For $H \cong K_{2}$, super $(a, d)$ - $H$-antimagic labelings are also called super $(a, d)$-edge-antimagic total labelings. For further information on super edge-magic labelings, one can see [9-12].

The super $(a, d)$-H-antimagic labeling is related to a super $d$-antimagic labeling of type $(1,1,0)$ of a plane graph which is the generalization of a face-magic labeling introduced by Lih [13]. Further information on super $d$-antimagic labelings can be found in [14-16].

In [17], Awais et al. proved the existence of $(a, d)$ - $C_{4}$-antimagic labeling of book graphs $B_{n}$ (for difference $d=0,1)$ and of its disjoint union. In this paper, we study the existence of super $(a, d)-C_{4}$-antimagic labeling of book graphs $B_{n}$ for differences $d=1,2,3, \ldots, 13$ and $n \geq 2$.

\section{Super Cycle Antimagic Labeling}

In this section, we discussed super $(a, d)$ - $C_{4}$-antimagicness of book graphs for difference $d=1,2,3, \ldots, 13$.

Let $K_{1, n}, n \geq 2$ be a complete bipartite graph on $n+1$ vertices. The book graph $B_{n}$ is a cartesian product of $K_{1, n}$ with $K_{2}$. i.e., $B_{n} \cong K_{1, n} \square K_{2}$. Clearly book graph $B_{n}$ admits $C_{4}$-covering. The book graph $B_{n}$ has the vertex set and edge set as

$$
\begin{gathered}
V\left(B_{n}\right)=\left\{y_{1}, y_{2}\right\} \cup \cup_{i=1}^{n}\left\{x_{(1, i)}, x_{(2, i)}\right\} \\
E\left(B_{n}\right)=\cup_{i=1}^{n}\left\{y_{1} x_{(1, i)}, y_{2} x_{(2, i)}, x_{(1, i)} x_{(2, i)}\right\} \cup\left\{y_{1} y_{2}\right\}
\end{gathered}
$$

respectively. It can be noted that $\left|V\left(B_{n}\right)\right|=2(n+1)$ and $\left|E\left(B_{n}\right)\right|=3 n+1$.

Every $C_{4}^{(j)}, 1 \leq j \leq n$ in $B_{n}$ has the vertex set: $V\left(C_{4}^{(j)}\right)=\left\{y_{1}, y_{2}, x_{(1, j)}, x_{(2, j)}\right\}$ and the edge set: $E\left(C_{4}^{(j)}\right)=$ $\left\{y_{1} y_{2}, y_{1} x_{(1, j)}, y_{2} x_{(2, j)}, x_{(1, j)} x_{(2, j)}\right\}$.

Under a total labeling $\xi$, the $C_{4}^{(j)}$-weights, $j=1, \ldots, n$, would be:

$$
\begin{aligned}
w_{\xi}\left(C_{4}^{(j)}\right) & =\sum_{v \in V\left(C_{4}^{(j)}\right)} \xi(v)+\sum_{e \in E\left(C_{4}^{(j)}\right)} \xi(e) . \\
& =\sum_{k=1}^{2}\left(\xi\left(y_{k}\right)+\xi\left(x_{(k, j)}\right)+\xi\left(y_{k} x_{(k, j)}\right)\right)+\xi\left(y_{1} y_{2}\right)+\xi\left(x_{(1, j)} x_{(2, j)}\right)
\end{aligned}
$$

Theorem 1. For any integer $n \geq 2$, the book graph $B_{n}$ admits super $(a, d)$ - $C_{4}$-antimagic labeling for differences $d=1,3, \ldots, 13$.

Proof. Under a labeling $\xi$, the set $\left\{y_{1}, y_{2}, y_{1} y_{2}\right\}$, would be labeled as:

$$
\begin{aligned}
\xi\left(y_{k}\right) & =k, \quad k=1,2 \\
\xi\left(y_{1} y_{2}\right) & =2(n+1)+1
\end{aligned}
$$

and therefore the partial sum of $w t_{\xi}\left(C_{4}^{(j)}\right)$ would be

$$
\xi\left(y_{1}\right)+\xi\left(y_{2}\right)+\xi\left(y_{1} y_{2}\right)=2(n+3) .
$$

For $d=1,3, \ldots, 9,13$

$$
\begin{aligned}
& \xi_{d}\left(x_{(k, j)}\right)= \begin{cases}2 j+1, & k=1 \\
2(j+1), & k=2\end{cases} \\
& \xi_{11}\left(x_{(k, j)}\right)= \begin{cases}2+j, & k=1 \\
n+2+j, & k=2\end{cases} \\
& \xi_{d}\left(x_{(1, j)} x_{(2, j)}\right)= \begin{cases}3 n+4-j, & d=1 \\
2 n+3+j, & d=3,5,7,9 \\
2 n+1+3 j, & d=11,13\end{cases}
\end{aligned}
$$




$$
\xi_{d}\left(y_{k} x_{(k, j)}\right)=\left\{\begin{array}{lll}
(k+3) n+4-j, & k=1,2 \quad d=1,3 \\
5 n+4-j, & k=1 \quad d=5 \\
3 n+3+j, & k=2 \quad d=5 \\
(k+2) n+3+j, & k=1,2 \quad d=7 \\
3 n+2 j+k+1, & k=1,2 \quad d=9 \\
3(n+j)-k, & k=1,2 \quad d=11 \\
3(n+j)+k-1, & k=1,2 \quad d=13
\end{array}\right.
$$

where indices $j$ are taken modulo $n$.

Clearly $\xi\left(V\left(B_{n}\right)\right)=\{1,2, \ldots, 2(n+1)\}$. Therefore $\xi$ is a super labeling together with $\xi\left(E\left(B_{n}\right)\right)=$ $\{2(n+1)+1,2(n+1)+2, \ldots, 5 n+3\}$ which shows $\xi$ is a total labeling.

Using (1) and (2), $w t_{\xi_{d}}\left(C_{4}^{(j)}\right)$ are:

$$
w t_{\xi_{d}}\left(C_{4}^{(j)}\right)= \begin{cases}14 n+21+j, & d=1 \\ 13 n+20+3 j, & d=3 \\ 12 n+19+5 j, & d=5 \\ 11 n+18+7 j, & d=7 \\ 10 n+17+9 j, & d=9 \\ 11 n+8+11 j, & d=11 \\ 10 n+11+13 j, & d=13\end{cases}
$$

Clearly $w t_{\tilde{\zeta}_{d}}\left(C_{4}^{(j)}\right)$ constitutes arithmetic progression and therefore book graphs are super $(a, d)$ - $C_{4}$-antimagic for $d=1,3, \ldots, 13$. This completes the proof.

Theorem 2. For any integer $n \geq 2$, the book graph $B_{n}$ admits super $(a, d)$-C $C_{4}$-antimagic labeling for differences $d=2,4, \ldots, 10$.

Proof. Case $n \equiv 0(\bmod 2)$

For $d=2,4,6,8$ the labeling $\xi$ for the set $\left\{y_{1}, y_{2}, y_{1} y_{2}\right\}$, would be labeled as:

$$
\begin{gathered}
\xi_{d}\left(y_{1}\right)=1 \\
\xi_{d}\left(y_{2}\right)=\frac{n}{2}+2 \\
\xi_{d}\left(y_{1} y_{2}\right)=2 n+3
\end{gathered}
$$

and therefore the partial sum of $w t_{\xi}\left(C_{4}^{(j)}\right)$ would be

$$
\xi_{d}\left(y_{1}\right)+\xi_{d}\left(y_{2}\right)+\xi_{d}\left(y_{1} y_{2}\right)=\frac{5 n}{2}+6
$$

The remaining set of elements has the labeling $\xi$ as:

$$
\xi_{d}\left(x_{(k, j)}\right)= \begin{cases}1+j, & k=1, j=1,2, \ldots, \frac{n}{2} \\ 2 j-\frac{n}{2}+1, & k=1, j=\frac{n}{2}+1, \ldots, n \\ \frac{n}{2}+2(1+j), & k=2, j=1,2, \ldots, \frac{n}{2} \\ n+2+j, & k=2, j=\frac{n}{2}+1, \ldots, n\end{cases}
$$




$$
\begin{gathered}
\xi_{d}\left(x_{(1, j)} x_{(2, j)}\right)= \begin{cases}2(n+1)+1+j, & d=2 \\
5 n+4-j, & d=4 \\
4 n+3+j, & d=6,8\end{cases} \\
\xi_{d}\left(y_{k} x_{(k, j)}\right)= \begin{cases}n(k+3)+4-j, & d=2 \\
n(k+1)+3+j, & d=4,6 \\
2(n+j)+k+1, & d=8\end{cases}
\end{gathered}
$$

For difference $d=10$ the labeling $\xi$ is defined as:

$$
\begin{aligned}
& \xi_{d}\left(y_{1}\right)=1 \\
& \xi_{d}\left(y_{2}\right)=\frac{3 n}{2}+2 \\
& \xi_{d}\left(y_{1} y_{2}\right)=2 n+3
\end{aligned}
$$

and the partial sum of $w t_{\xi}\left(C_{4}^{(j)}\right)$ would be:

$$
\begin{gathered}
\xi_{d}\left(y_{1}\right)+\xi_{d}\left(y_{2}\right)+\xi_{d}\left(y_{1} y_{2}\right)=\frac{7 n}{2}+6 \\
\xi_{d}\left(x_{(k, j)}\right)= \begin{cases}2 j+1, & k=1, j=1,2, \ldots, \frac{n}{2} \\
2 j-n, & k=1, j=\frac{n}{2}+1, \ldots, n \\
\frac{3 n}{2}+2-j, & k=2, j=1,2, \ldots, \frac{n}{2} \\
\frac{5 n}{2}+3-j, & k=2, j=\frac{n}{2}+1, \ldots, n\end{cases} \\
\xi_{d}\left(x_{(1, j)} x_{(2, j)}\right)=2 n+1+3 j \\
\xi_{d}\left(y_{k} x_{(k, j)}\right)=2 n+(k+1)+3 j, \quad k=1,2
\end{gathered}
$$

Clearly $\xi\left(V\left(B_{n}\right)\right)=\{1,2, \ldots, 2(n+1)\}$. Therefore $\xi$ is a super labeling and together with $\xi\left(E\left(B_{n}\right)\right)=$ $\{2(n+1)+1,2(n+1)+2, \ldots, 5 n+3\}$ which shows $\xi$ is a total labeling.

Using (1), (3) and (4), $w_{\xi}\left(C_{4}^{(j)}\right)$ are:

$$
w_{\xi_{d}}\left(C_{4}^{(j)}\right)= \begin{cases}14 n+20+2 j, & d=2 \\ 13 n+19+4 j, & d=4 \\ 12 n+18+6 j, & d=6 \\ 11 n+17+8 j, & d=8 \\ 11 n+15+10 j, & d=10\end{cases}
$$

Therefore $w t_{\xi_{d}}\left(C_{4}^{(j)}\right)$ constitutes arithmetic progression for differences $d=2,4, \ldots, 10$ when $n \equiv 0(\bmod 2)$.

Case $n \equiv 1(\bmod 2)$

For the set $\left\{y_{1}, y_{2}, y_{1} y_{2}\right\}$, labeling $\xi$ would be:

$$
\begin{gathered}
\xi_{d}\left(y_{1}\right)=1 \\
\xi_{d}\left(y_{2}\right)=n+2 \\
\xi_{d}\left(y_{1} y_{2}\right)=2 n+3
\end{gathered}
$$

and therefore the partial sum of $w t_{\tilde{\xi}}\left(C_{4}^{(j)}\right)$ would be

$$
\xi_{d}\left(y_{1}\right)+\xi_{d}\left(y_{2}\right)+\xi_{d}\left(y_{1} y_{2}\right)=3(n+2)
$$


For differences $d=2,4,6,10$

$$
\xi_{d}\left(x_{(k, j)}\right)= \begin{cases}2 j, & k=1, j=1,2, \ldots, \frac{n+1}{2} \\ 2 j-n, & k=1, j=\frac{n+1}{2}+1, \ldots, n \\ 3\left(\frac{n+1}{2}\right)+2-j, & k=2, j=1,2, \ldots, \frac{n+1}{2} \\ 5\left(\frac{n+1}{2}\right)+1-j, & k=2, j=\frac{n+1}{2}+1, \ldots, n\end{cases}
$$

and for differences $d=8$

$$
\xi_{d}\left(x_{(k, j)}\right)= \begin{cases}n+2-2 j, & k=1, j=1,2, \ldots, \frac{n-1}{2} \\ 2(n+1)-2 j, & k=1, j=\frac{n+1}{2}, \ldots, n \\ 3\left(\frac{n+1}{2}\right)+1+j, & k=2, j=1,2, \ldots, \frac{n-1}{2} \\ \frac{n+1}{2}+2+j, & k=2, j=\frac{n+1}{2}, \ldots, n\end{cases}
$$

For differences $d=2,4, \ldots, 10$, the set of edges has the labeling $\xi$ defined as:

$$
\begin{gathered}
\xi_{d}\left(x_{(1, j)} x_{(2, j)}\right)= \begin{cases}5 n+4-j, & d=2 \\
4 n+3+j, & d=4,6 \\
2 n+3+3 j, & d=8,10\end{cases} \\
\xi_{d}\left(y_{k} x_{(k, j)}\right)= \begin{cases}n(k+1)+3+j, & k=1,2, \quad d=2,4 \\
2(n+j)+k+1, & k=1,2, \quad d=6 \\
2 n+k+3 j, & d=8,10\end{cases}
\end{gathered}
$$

Clearly $\xi\left(V\left(B_{n}\right)\right)=\{1,2, \ldots, 2(n+1)\}$. Therefore $\xi$ is a super labeling together with $\xi\left(E\left(B_{n}\right)\right)=\{2(n+$ $1)+1,2(n+1)+2, \ldots, 5 n+3\}$ which shows $\xi$ is a total labeling.

Using (1) and (5), $w t_{\xi}\left(C_{4}^{(j)}\right)$ are:

$$
w t_{\xi_{d}}\left(C_{4}^{(j)}\right)= \begin{cases}\frac{27 n+33}{2}+2 j, & d=2 \\ \frac{25 n+31}{2}+4 j, & d=4 \\ \frac{23 n+29}{2}+6 j, & d=6 \\ \frac{21 n+27}{2}+8 j, & d=8 \\ \frac{19 n+25}{2}+4 j, & d=10\end{cases}
$$

Therefore $w t_{\xi_{d}}\left(C_{4}^{(j)}\right)$ constitute arithmetic progression for differences $d=2,4, \ldots, 10$ when $n \equiv 1(\bmod 2)$. Hence book graphs are super $(a, d)-C_{4}$-antimagic for $d=2,4, \ldots, 10$. This completes the proof.

Theorem 3. For any integer $n \geq 2$, the book graph $B_{n}$ admits super $(a, 12)-C_{4}$-antimagic labeling.

\section{Proof. Case $n \equiv 0(\bmod 2)$}

Under a labeling $\xi$, the set $\left\{y_{1}, y_{2}, y_{1} y_{2}\right\}$, would be labeled as:

$$
\begin{aligned}
\xi_{12}\left(y_{1}\right) & =1 \\
\xi_{12}\left(y_{2}\right) & =\frac{n+4}{2} \\
\xi_{12}\left(y_{1} y_{2}\right) & =2 n+3
\end{aligned}
$$


and therefore the partial sum of $w t_{\xi}\left(C_{4}^{(j)}\right)$ would be

$$
\begin{gathered}
\xi_{12}\left(y_{1}\right)+\xi_{d}\left(y_{2}\right)+\xi_{d}\left(y_{1} y_{2}\right)=\frac{5 n+12}{2} \\
\xi_{12}\left(x_{(k, j)}\right)= \begin{cases}1+j & k=1, j=1,2, \ldots, \frac{n}{2} \\
2 j+1-\frac{n}{2} & k=1, j=\frac{n}{2}+1, \ldots, n \\
\frac{n}{2}+2(1+j), & k=2, j=1,2, \ldots, \frac{n}{2} \\
n+2+j, & k=2, j=\frac{n}{2}+1, \ldots, n\end{cases} \\
\xi_{12}\left(y_{k} x_{(k, j)}\right)=2(n+k)+3 j-1 \\
\xi_{12}\left(x_{(1, j)} x_{(2, j)}\right)=2(n+1)+3 j
\end{gathered}
$$

where indices $j$ are taken modulo $n$.

$\underline{\text { Case } n \equiv 1(\bmod 2)}$

Under a labeling $\xi$, the set $\left\{y_{1}, y_{2}, y_{1} y_{2}\right\}$, would be labeled as:

$$
\begin{gathered}
\xi_{12}\left(y_{k}\right)=\frac{3}{2}(n-1)+2 k \\
\xi_{12}\left(y_{1} y_{2}\right)=2 n+3
\end{gathered}
$$

and therefore the partial sum of $w t_{\xi}\left(C_{4}^{(j)}\right)$ would be

$$
\begin{gathered}
\xi_{12}\left(y_{1}\right)+\xi_{d}\left(y_{2}\right)+\xi_{d}\left(y_{1} y_{2}\right)=5 n+6 \\
\xi_{12}\left(x_{(k, j)}\right)= \begin{cases}j & k=1, j=1,2, \ldots, \frac{n+1}{2} \\
2 j-\frac{n+3}{2} & k=1, j=\frac{n+1}{2}+1, \ldots, n \\
\frac{n+1}{2}+2 j, & k=2, j=\frac{n}{2}+1, \ldots, n \\
n+2+j, & k=2, j=\frac{n}{2}+1, \ldots, n\end{cases} \\
\xi_{12}\left(y_{k} x_{(k, j)}\right)=2 n+k+3 j \quad k=1,2 \\
\xi_{12}\left(x_{(1, j)} x_{(2, j)}\right)=2 n+3(1+j)
\end{gathered}
$$

where indices $j$ are taken modulo $n$.

Clearly $\xi\left(V\left(B_{n}\right)\right)=\{1,2, \ldots, 2(n+1)\}$. Therefore $\xi$ is a super labeling together with $\xi\left(E\left(B_{n}\right)\right)=\{2(n+$ 1) $+1,2(n+1)+2, \ldots, 5 n+3\}$ which shows $\xi$ is a total labeling.

Using (1), (6) and (7), $w t_{\tilde{\xi}}\left(C_{4}^{(j)}\right)$ are:

$$
w t_{\xi_{12}}\left(C_{4}^{(j)}\right)=\left\{\begin{array}{lll}
3(3 n+5)+12 j & n \equiv 0 & (\bmod 2) \\
\frac{23 n+25}{2}+12 j & n \equiv 1 \quad(\bmod 2)
\end{array}\right.
$$

Hence book graphs are super $(a, 12)-C_{4}$-antimagic. This completes the proof.

Acknowledgments: The author wishes to express his profound gratitude to the reviewers for their useful comments on the manuscript.

Author Contributions: All authors contributed equally to the writing of this paper. All authors read and approved the final manuscript.

Conflicts of Interest: The authors declare no conflict of interest. 


\section{References}

[1] Gutiérrez, A., \& Lladó, A. (2005). Magic coverings. Journal of Combinatorial Mathematics and Combinatorial Computing, 55(2005), 43-56.

[2] Lladó, A., \& Moragas, J. (2007). Cycle-magic graphs. Discrete Mathematics, 307(23), 2925-2933.

[3] Ngurah, A. A. G., Salman, A. N. M., \& Susilowati, L. (2010). H-supermagic labelings of graphs. Discrete Mathematics, 310(8), 1293-1300.

[4] Maryati, T. K., Baskoro, E. T., \& Salman, A. N. M. (2008). $P_{h^{-}}$(super)magic labelings of some trees. Journal of Combinatorial Mathematics and Combinatorial Computing, 65, 198-204.

[5] Jeyanthi, P., \& Selvagopal, P. (2010). More classes of H-supermagic graphs. International Jornal of Algorithms, Computing and Mathematics, 3(1), 93-108.

[6] Maryati, T. K., Salman, A. N. M., \& Baskoro, E. T. (2013). Supermagic coverings of the disjoint union of graphs and amalgamations. Discrete Mathematics, 313(4), 397-405.

[7] Inayah, N., Salman, A. N. M., \& Simanjuntak, R. (2009). On (a,d)-H-antimagic coverings of graphs. Journal of Combinatorial Mathematics and Combinatorial Computing, 71, 273-281.

[8] Inayah, N., Simanjuntak, R., Salman, A. N. M., \& Syuhada, K. I. A. (2013). Super (a,d)-H-antimagic total labelings for shackles of a connected graph H. Australasian Journal of Combinatorics, 57, 127-138.

[9] Wallis, W. D. (2001). Magic graphs. Birkhäuser, New York.

[10] Bača, M., Lin, Y. Q., Muntaner-Batle, F. A., \& Rius-Font, M. (2009). Strong labelings of linear forests. Acta Mathematica Sinica, English Series, 25(12), 1951-1964.

[11] Baca, M., \& Miller, M. (2008). Super edge-antimagic graphs: A wealth of problems and some solutions. Universal-Publishers.

[12] Figueroa-Centeno, R. M., Ichishima, R., \& Muntaner-Batle, F. A. (2001). The place of super edge-magic labelings among other classes of labelings. Discrete Mathematics, 231(1-3), 153-168.

[13] Lih, K. W. (1983). On magic and consecutive labelings of plane graphs. Utilitas Math, 24, 165-197.

[14] Bača, M., Miller, M., Phanalasy, O., \& Semaničová-Feňovčíková, A. (2010). Super d-antimagic labelings of disconnected plane graphs. Acta Mathematica Sinica, English Series, 26(12), 2283-2294.

[15] Bača, M., Brankovic, L., Semaničová-Feňovčíková, A., Labelings of plane graphs containing Hamilton path, Acta Math. Sinica - English Series, 2011, 27(4), 701-714.

[16] Bača, M., Numan, M., \& Shabbir, A. (2013). Labelings of type $(1,1,1)$ for toroidal fullerenes. Turkish Journal of Mathematics, 37(6), 899-907.

[17] Umar, M. A., Javed, M. A., Hussain, M., \& Ali, B. R. (2018).Super ( $a, d)$ - $C_{4}$-antimagicness of book graphs. Open Journla of Mathematical Sciences, 2, 115-121.

(C) 2019 by the authors; licensee PSRP, Lahore, Pakistan. This article is an open access article distributed under the terms and conditions of the Creative Commons Attribution (CC-BY) license (http://creativecommons.org/licenses/by/4.0/). 